\title{
SCHEMES AND OPTIMIZATION OF GAS FLOWING INTO THE ION SOURCE AND THE NEUTRALIZER OF THE DIII-D NEUTRAL BEAM SYSTEMS
}

\author{
by \\ R.M. HONG and H.K. CHIU
}

This is a preprint of a paper to be presented at the 18th IEEE/NPSS Symposium on Fusion Engineering, October 25-29, 1999, in Albuquerque, New Mexico, and to be published in the Proceedings.

\author{
Work supported by \\ the U.S. Department of Energy \\ under Contract No. DE-AC03-99ER54463
}




\section{DISCLAIMER}

This report was prepared as an account of work sponsored by an agency of the United States Government. Neither the United States Government nor any agency thereof, nor any of their employees, make any warranty, express or implied, or assumes any legal liability or responsibility for the accuracy, completeness, or usefuiness of any information, apparatus, product, or process disclosed, or represents that its use would not infringe privately owned rights. Reference herein to any specific commercial product, process, or service by trade name, trademark, manufacturer, or otherwise does not necessarily constitute or imply its endorsement, recommendation, or favoring by the United States Government or any agency thereof. The views and opinions of authors expressed herein do not necessarily state or reflect those of the United States Government or any agency thereof. 


\section{DISCLAIMER}

Portions of this document may be illegible in electronic image products. Images are produced from the best available original document. 


\title{
R.M. HONG AND H.K. CHIU \\ Schemes and Optimization of Gas Flowing Into the Ion Source and the Neutralizer of the DIII-D Neutral Beam Systems*
}

\author{
R.M. Hong and H.K. Chiu \\ General Atomics, P.O. Box 85608, San Diego, California 92186-5608
}

\begin{abstract}
Performance comparisons of a DIII-D neutral beam ion source operated with two different schemes of supplying neutral gas to the arc chamber were performed. Superior performance was achieved when gas was puffed into both the arc chamber and the neutralizer with the gas flows optimized as compared to supplying gas through the neutralizer alone. To form a neutral beam, ions extracted from the arc chamber and accelerated are passed through a neutralizing cell of gas. Neutral gas is commonly puffed into the neutralizing cell to supplement the residual neutral gas from the arc chamber to obtain maximum neutralization efficiency. However, maximizing neutralization efficiency does not necessarily provide the maximum available neutral beam power, since high levels of neutral gas can increase beam loss through collisions and cause larger beam divergence. Excessive gas diffused from the neutralizer into the accelerator region also increases the number of energetic particles (ions and secondary electrons from the accelerator grid surfaces) deposited on the accelerator grids, increasing the possibility of overheating. We have operated an ion source with a constant optimal gas flow directly into the arc chamber while gas flow into the neutralizer was varied. Neutral beam power available for injecting into plasmas was obtained based on the measured data of beam energy, beam current, beam transmission, beam divergence, and neutralization efficiency for various neutralizer gas flow rates. We will present the results of performance comparison with the two gas puffing schemes, and show steps of obtaining the maximum available beam power and determining the optimum neutralizer gas flow rate.
\end{abstract}

\section{INTRODUCTION}

Performance of the ion source and neutralization efficiency of energetic ions are the two dominant factors which determine the injected beam power of a neutral beam system used as an auxiliary plasma heating system in fusion research. The scheme of puffing gas into the ion source and the neutralizer cell directly affects the ion source performance and neutralization efficiency. Puffing gas directly into the arc chamber of an ion source is the most commonly used scheme of supplying gas to an ion source for arc discharge. It requires electrical insulation between the gas system, normally at ground potential, and the ion source which is at high potential ( $80 \mathrm{kV}$ for the DIII-D ion source). For insulation purpose, non-metalic tubing is used to deliver gas to the arc chamber. This scheme may not be practical for an ion source operated either in highly radioactive gas, such as tritium, due to safety concerns (non-metal tubing has a higher risk of failure and leakage) or at a very high beam energy, which may have insulation difficulities. An alternate scheme is introducing gas into the neutralizer of the beamline and letting the gas diffuse through the accelerator into the arc chamber. It requires no electrical insulation since the neutralizer and the gas line are both at ground potential. There are advantages and disadvantages for each of the two schemes; however, we are interested in the performance of the ion source with each gas delivery scheme. Introducing neutral gas into the neutralizer is necessary to obtain higher neutralization efficiency of the energetic ion beam, but it also increases the beam divergence, producing more beam loss along the beamline, and it also increases beam loss within the accelerator region. It is then, necessary to optimize the amount of neutral gas puffed into the neutralizer by considering and weighing all these factors.

\section{COMPARISON OF ION SOURCE PERFORMANCE WITH TWO SCHEMES OF GAS PUFFING}

\section{A. Operating Parameters}

DIII-D neutral beam ion source consists of an arc chamber and an accelerator with four grids (plasma, gradient, suppressor, and exit grid). To compare source performance, we fixed the following operating parameters for the two gas puffing schemes: voltage applied to the filaments of the arc chamber was constant at $6.8 \mathrm{~V}$, the Langmuir probe signal which is used to measure and regulate the density of the arc discharge inside the arc chamber was set at $1.63 \mathrm{~V}$, beam energy was set at $70 \mathrm{keV}$, and source was operated in deuterium gas with beam pulse length up to $2.5 \mathrm{~s}$.

\section{B. Test Procedures}

For each gas delivery scheme, arc power required to produce a constant arc discharge density was measured for each gas flow rate. Repeat measurements were taken for consistency comparison. To compare the performance of the ion source when ions were extracted from the arc chamber and accelerated to high energy by the accelerator, the gas flow rate for each gas delivery scheme was chosen such that identical arc power was required to obtain a constant arc discharge density.

\section{Performance Comparison}

Figure 1 shows clearly that less arc power was required and discharges were more consistent when gas was puffed directly into the arc chamber. Comparing the gas flow required for each scheme when $75 \mathrm{~kW}$ arc power was needed for the discharge we find that the rate of gas flowing into the

\footnotetext{
*Work supported by U.S. Department of Energy under Contract No. DE-AC03-99ER54463.
} 


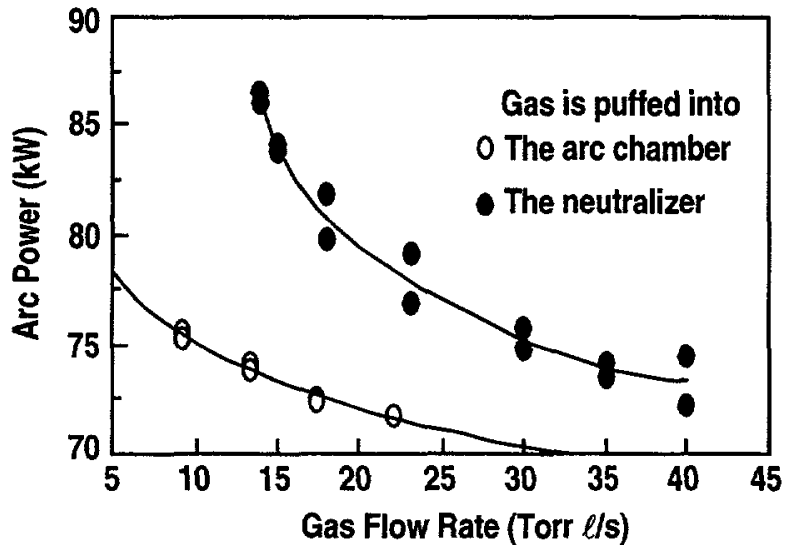

Fig. 1. Arc power required to sustain a constant arc discharge density for two schemes of gas puffing.

neutralizer was about three times that of the case when gas was puffed directly into the arc chamber. We then chose this three to one gas flow rate ratio and operated the ion source to extract and accelerate the ion beams. The ion source was first operated with 10 Torr $\ell / s$ gas flowing directly into the arc chamber. The ion source was then operated with $30 \mathrm{Torr} \ell / \mathrm{s}$ gas flowing into the neutralizer, but no gas was flowing into the arc chamber. The measured beam currents for various beam pulse length were plotted in Fig. 2. A very slight increase in the beam current with longer beam pulses is due to the intrinsic characteristic (called the effect of backstreaming electrons) of the ion source. The effect has been suppressed by the regulation of arc discharge density inside the arc chamber. It is shown in Fig. 2 that there is a difference of about $10 \%$ in the beam current for the two schemes of gas puffing, indicating a larger beam loss within the accelerator region when gas was flowing into the neutralizer. This is also confirmed by the much larger gradient grid current (more than six to one ratio, shown in Fig. 3). All of these data concluded that the ion source performed much better, as measured by the characteristics of beams produced by the source, when gas was puffed directly into the arc chamber for arc discharges. We should also point out that when we attempted to operate the ion source with gas flowing into the neutralizer at a rate of

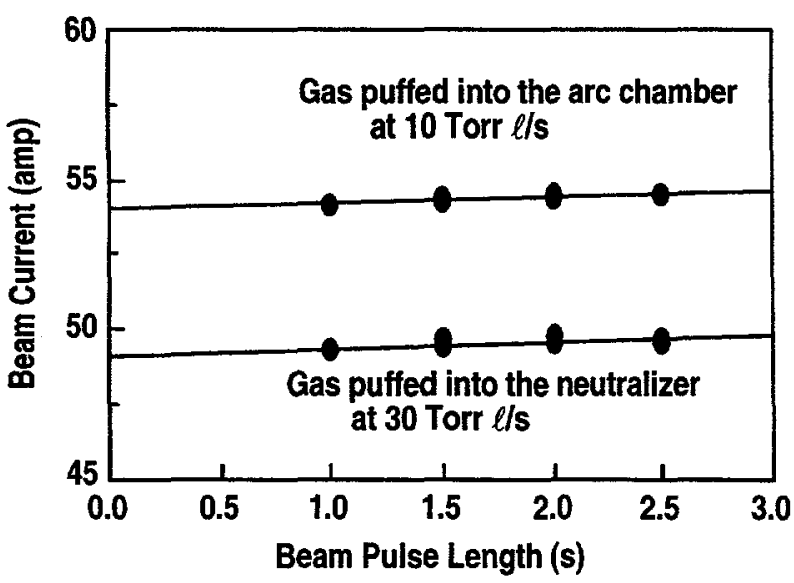

Fig. 2. Beam current for the two schemes of gas puffing.

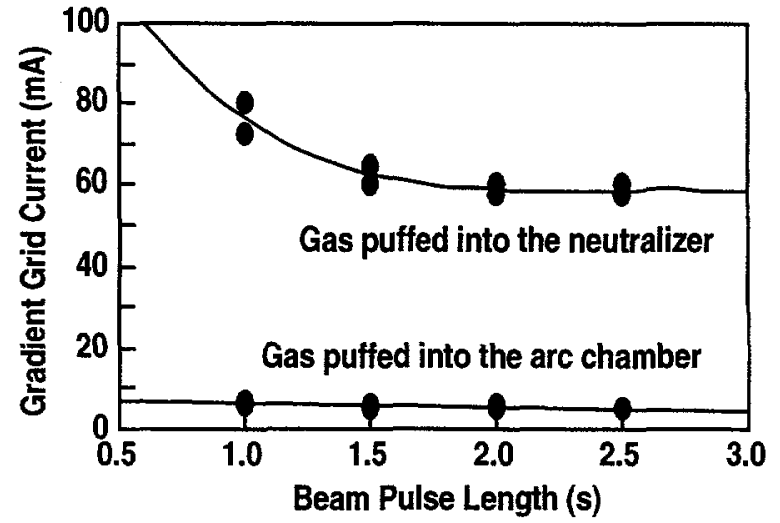

Fig. 3. Gradient grid current for the two schemes of gas puffing.

25 Torr $\ell / \mathrm{s}$ or less, the operation became unstable with a decaying beam current during the beam pulsing, indicating a severe gas starvation condition. Even a gas flow of 10 Torr $\ell / \mathrm{s}$ puffed directly into the arc chamber is marginally enough to avoid a gas starvation condition.

\section{OPTIMIZATION OF GAS FLOWING INTO THE NEUTRALIZER}

To experimentally demonstrate the procedures of optimizing the neutralizer gas flow, we had set the gas flow directly into the arc chamber at a constant rate of 15 Torr $\ell / \mathrm{s}$ while operating the ion source with several rates of gas puffing into the neutralizer.

\section{A. Beam and Gradient Grid Currents}

The beam current of a neutral beam system is the measurement of the amount of ions coming out of an ion source after they are extracted from the arc chamber and accelerated by the accelerator. The gradient grid current measures the amount of energetic particles deposited on the gradient grid of the accelerator, and is the most sensitive indication of beam optics for "tuning" the ion source [1]. It is always desirable to operate an ion source with the smallest gradient grid current as it represents operating the ion source at the optimum beam perveance (best beam optics) and avoids possible accelerator grid overheating. Figure 4 plots the beam and gradient grid currents as functions of the neutralizer gas flow rate. As more gas was puffed into the neutralizer, larger gradient grid currents and smaller beam currents were the results of increased loss of ion beam within the accelerator region due to collisions between energetic ions and neutral particles. Note that the rate of change of the gradient grid current is much larger than that of the beam current, confirming that the gradient grid current is the most sensitive indication of the beam optics.

\section{B. Neutralization Efficiency and Beam Loss Between the Ion Source and the Calorimeter}

The temperature change of the calorimeter, which is used as the beam dump during ion source conditioning, tuning, and calibrations, was used to calculate the neutralization 


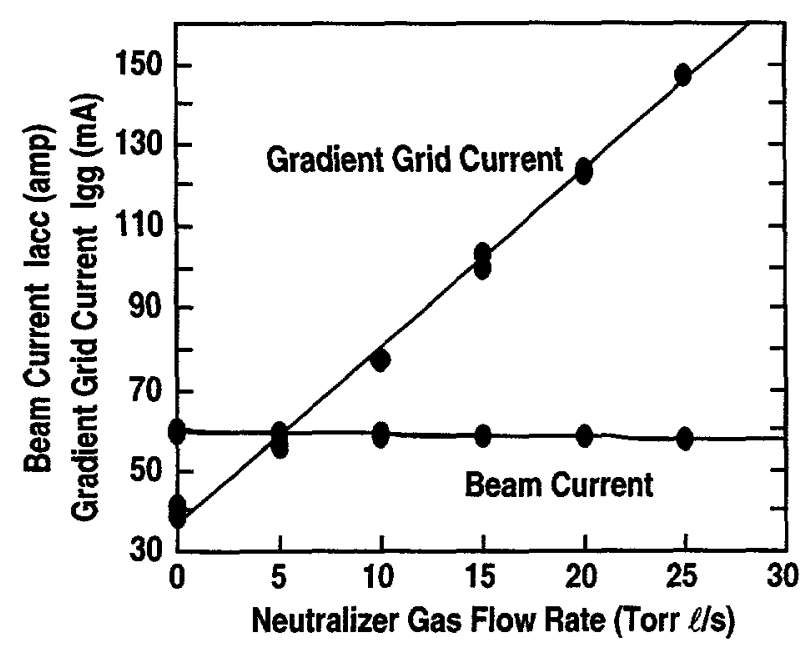

Fig. 4. Beam and gradient grid currents as function of the neutralizer gas flow rate.

efficiency. Thermocouples were embedded in the calorimeter to measure its temperature change. Residual energetic ions (ions passed through the neutralizer, but are not neutralized) are bent into the ion dump and miss the calorimeter when the bending magnet is energized. The ratio of the calorimeter thermocouple temperature, which rises with or without the bending magnet being energized, can then be used as one method of measuring the neutralization efficiency. Figure 5 shows the measured results, indicating that the neutralization efficiency continues to go higher with more gas flowing into the neutralizer, approaches an asymptoic value. However, some of the beam will lose to the beamline collimators before reaching the calorimeter. The amount of beam reaching the calorimeter is dependent of the beam perveance which is a function of the gas flowing into the neutralizer. Figure 6 shows the beam transmission efficiency from the ion source to the calorimeter. The amount of neutral beam reaches the calorimeter can now be expressed in terms of the product of the measured beam current, the beam transmission efficiency and the neutralization efficiency.

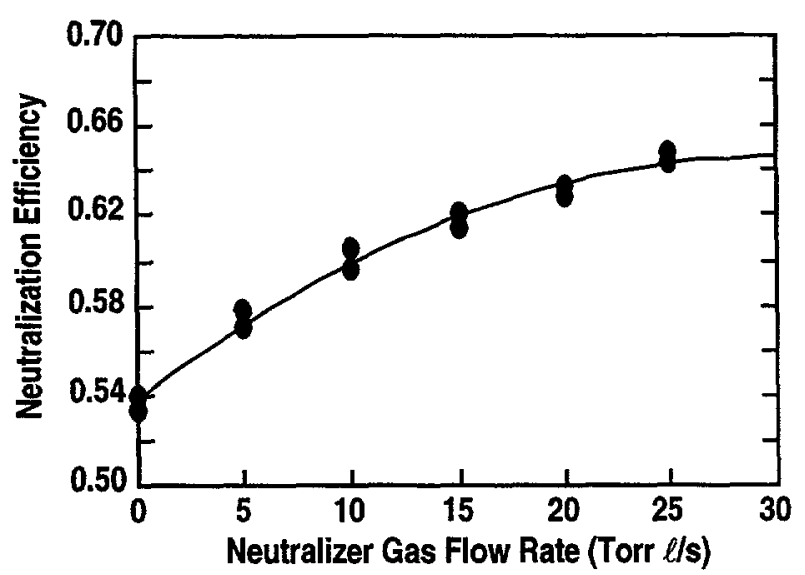

Fig. 5. Neutralization efficiency as function of the neutralizer gas flow rate.

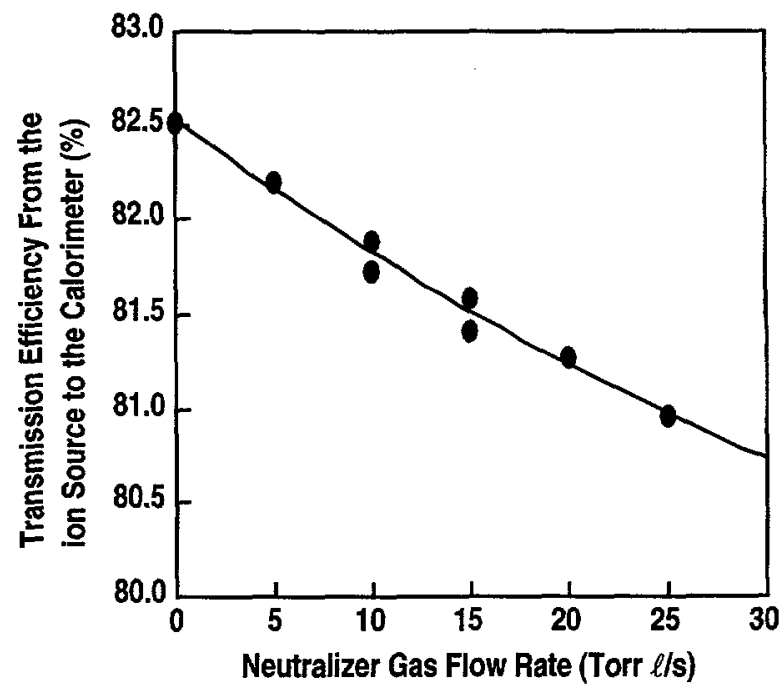

Fig. 6. Beam transmission efficiency from the ion source to the calorimeter.

\section{Beam Divergence and Beam Transmission Efficiency}

Readings of the calorimeter thermocouples array were also used to obtain the beam profiles (in the two dimensional plan normal to the beam path) and the beam divergent angles [2]. Figure 7 shows that beam divergent angles increased as more gas was puffed into the neutralizer. This is consistent with the observation that the gradient grid current increases and the beam current decreases when more gas was puffed into the neutralizer, and it is all due to increased collisions between the beam and the neutral gas particles. Using the beam profiles, the divergent angles, and the beamline hardware geometry, we calculated the beam transmission efficiency between the calorimeter and the tokamak vessel. Results are shown in Fig. 8.

\section{Optimum Neutralizer Gas Flow Rate}

Combining transmission efficiency between the calorimeter and the tokamak vessel and the amount of neutral

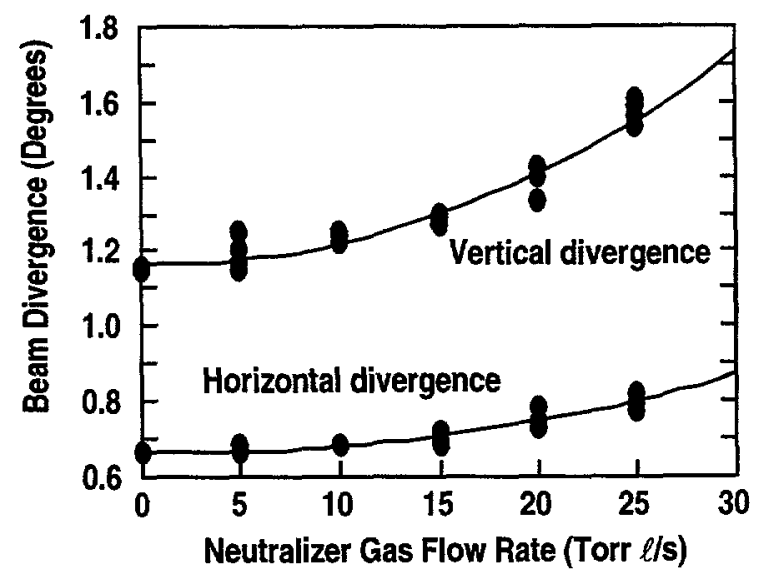

Fig. 7. Beam divergence increases as more gas flows into the neutralizer. 


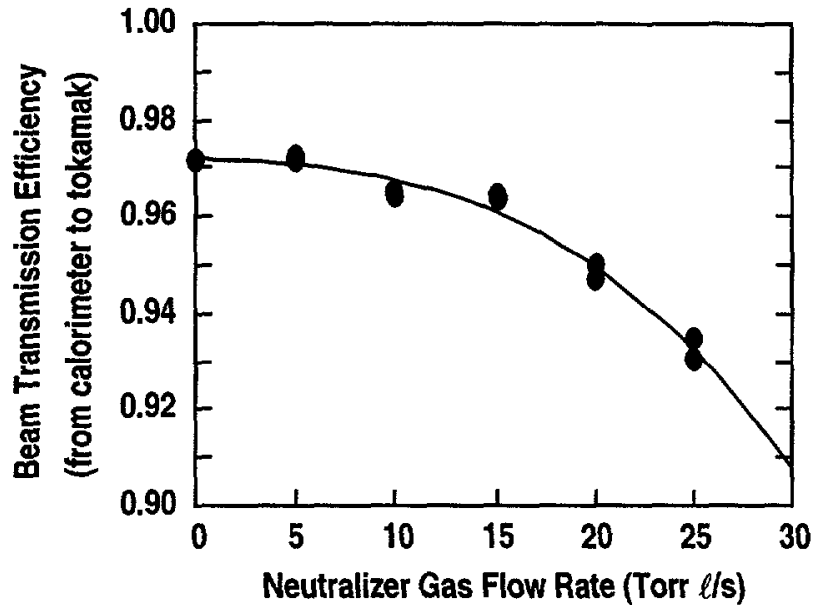

Fig. 8. Beam transmission efficiency decreases as more gas flows into the neutralizer.

beam reaches the calorimeter, we obtained the neutral beam current (shown in Fig. 9) "injected" into the tokamak vessel for various neutralizer gas flow rates. The actual injected neutral beam power is this "injected" beam current times the beam energy. Figure 9 shows that the injected neutral beam current (and beam power) reached the maximum value when the gas flowing into the neutralizer was about $15 \mathrm{Torr} \ell / \mathrm{s}$ and started to decrease slowly as more gas was puffed into the neutralizer. Before claiming that 15 Torr $\ell / \mathrm{s}$ is the optimum neutralizer gas flow rate, we need to see if the gradient grid current at this neutralizer gas flow rate is within the range for safe operation of the ion source. To protect the gradient grid from damage due to overheating, we set the goal of operating the ion source with the gradient grid current as low as possible, and ion source operation will be terminated if the gradient grid current reaches $200 \mathrm{~mA}$. Figure 4 shows that the gradient grid current, with 15 Torr $\ell / s$ neutralizer gas flow, is about $100 \mathrm{~mA}$ which is well below our operation limitation of $200 \mathrm{~mA}$. We can now claim that 15 Torr $\ell / \mathrm{s}$ is the optimum gas flow for the neutralizer when $15 \mathrm{Torr} \ell / \mathrm{s}$ of gas is puffed directly into the arc chamber.

\section{DISCUSSION AND CONCLUSIONS}

We have shown that the ion source achieved superior performance when gas was puffed directly into the arc chamber for arc discharge, and a relatively high gas flow was required to obtain stable beam operation when gas was puffed only into the neutralizer. However, it is necessary to puff gas into the neutralizer to achieve high neutralization efficiency even when puffing gas directly into the arc chamber for ion source operation. We have then described an experimental procedure to identify the optimum rate of gas flowing into the neutralizer for achieving maximum available neutral beam

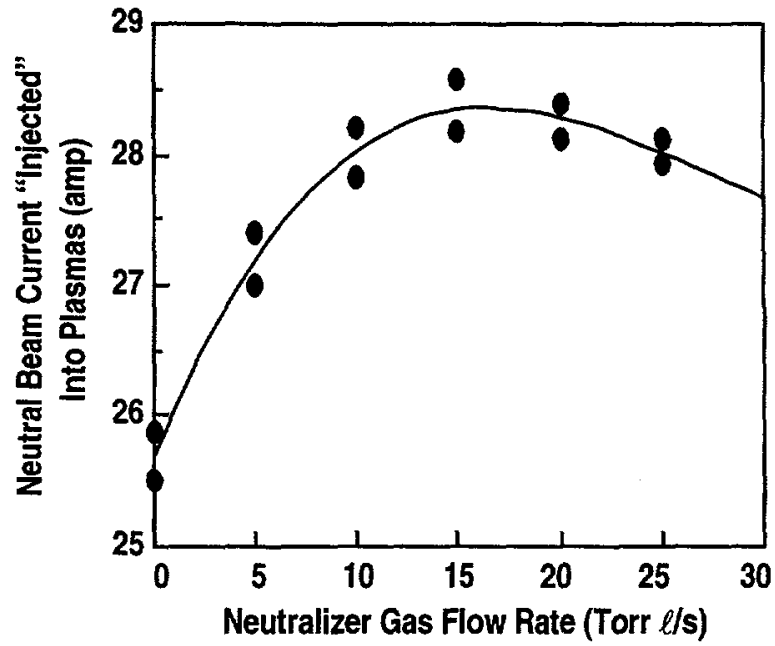

Fig. 9. Injected neutral beam current as function of the neutralizer gas flow rate.

power for injecting into plasmas of a tokamak. In deciding the optimum gas flow rate, safe operation of the ion source should also be considered. We note here that the value of the optimum neutralizer gas flow rate in our experiment was obtained for a specific and fixed rate of gas flowing directly into the arc chamber. The optimum neutralizer gas flow rate will vary if the amount of gas flowing directly into the arc chamber changes, since the residual gas inside the arc chamber will diffuse into the accelerator and the neutralizer regions, affecting the beam optics and beam transmission efficiency. The arc discharge density inside the arc chamber varies to meet the needs of operating the ion source at various beam energies, and higher arc discharge density will yield a lesser amount of residual gas inside the arc chamber. Therefore, the optimum neutralizer gas flow rate will also depend on the beam energy. Ideally, a series of tests need to be performed to obtain the optimum neutralizer and arc chamber gas flow rates for various beam energies.

\section{ACKNOWLEDGEMENT}

The authors would like to thank Bonnie Scoville for operating the ion source and helping with the data collection.

\section{REFERENCE}

[1] R. Hong, et al., "Operational Experience with the DIII-D Neutral Beam $12 \mathrm{~cm} \times 48 \mathrm{~cm}$ Common Long Pulse Source," Proc. 12th Symp. on Fusion Engineering, IEEE, Monterey, 2, 1133 (1987).

[2] H.K. Chiu, "Measurement of Neutral Beam Profiles at DIII-D," Proc. 13th Top. Mtg. on the Technology of Fusion Energy, Fusion Technology Vol. 34 (3), part 2 564 (1998). 